\title{
Incidence and treatment outcomes of secondary epiretinal membrane following intravitreal injection for diabetic macular edema
}

\author{
Yong Koo Kang (D, Han Sang Park, Dong Ho Park (i) \& Jae Pil Shin (i) * \\ The purpose of this study was to investigate the incidence of secondary epiretinal membrane (ERM) \\ after intravitreal injection and the effect of ERM on visual acuity and central macular thickness (CMT) in \\ patients with diabetic macular edema (DME). We included 147 eyes of 95 patients over 18 years old who \\ were diagnosed with DME from 2012 to 2016, treated with intravitreal injection, and followed-up more \\ than 24 months. Mean CMT in the ERM group was significantly thicker than in the non-ERM group after \\ $9,12,18$, and 24 months. Secondary ERM developed in 9.5\% of patients during follow-up. Compared \\ to other agents, the incidence of secondary ERM was significantly higher after intravitreal injection of \\ dexamethasone implant. Among patients in the ERM group, the mean decrease of CMT between pre- \\ injection and 2 weeks post-injection was significantly less after secondary ERM formation than before \\ ERM formation. Secondary ERM formation was significantly associated with the number of intravitreal \\ injections and the use of dexamethasone implant. Therefore, secondary ERM develops more frequently \\ as the number of intravitreal injections increases and after intravitreal dexamethasone implant \\ injection. The therapeutic effects of intravitreal injections for DME patients decrease after secondary \\ ERM formation.
}

Diabetic macular edema (DME) remains the most common cause of visual loss among diabetic retinopathy (DR) patients ${ }^{1}$. DME occurs following the breakdown of the blood-retinal barrier due to damage to vascular endothelial cell tight junctions and the loss of pericytes in retinal capillaries. DME is also caused by up regulation of increased inflammatory cytokines, such as chemokines, interleukin-6, interleukin-8, prostaglandins, and vascular endothelial growth factor (VEGF) induced by retinal tissue hypoxia ${ }^{1-3}$. Thus, intravitreal injections of anti-VEGF and steroids have been considered the standard management of DME and have been shown to improve clinical outcomes $^{4-6}$.

Epiretinal membrane (ERM) is a pathology caused by a fibrocellular proliferation on the internal limiting membrane (ILM) followed by a cellular contraction and can occur secondary to $\mathrm{DR}^{7}$. Glial cells in ERM produce various cytokines and growth factors, which stimulate pathogenic neovascularization mediated by VEGF. VEGF and its receptors are localized to both vascular and avascular ERM, and VEGF may contribute to the progression of $\mathrm{DME}^{9}$. Moreover, ERM may act as a physical barrier and decrease drug penetration after intravitreal injections of anti-VEGF or steroids in DME treatment ${ }^{10}$

We investigated the incidence of secondary ERM and the effect of ERM on visual acuity and central macular thickness (CMT) among DME patients who received intravitreal injections. We also analyzed parameters contributing to secondary ERM formation following intravitreal injection.

\section{Results}

Demographics of patient. One hundred thirty-four patients were initially enrolled, but 39 patients were excluded. Thus, a total of 95 patients (147 eyes) were enrolled in this study. The demographic and clinical characteristics of all patients are summarized in Table 1.

Among the 95 patients (147 eyes), 46 were male, and 49 were female. The mean age of all patients was $59.7 \pm 12.7$ years, and the duration of diabetes mellitus was $15.3 \pm 8.6$ years. Thirty-five patients had hypertension, 


\begin{tabular}{|c|c|c|}
\hline Characteristics & Value & $P$ value \\
\hline \multicolumn{3}{|l|}{ Number of eyes, n (\%) } \\
\hline OD & $75(51.0 \%)$ & \\
\hline OS & $72(49.0 \%)$ & \\
\hline \multicolumn{3}{|l|}{ Sex, n (\%) } \\
\hline Male & $46(48.4 \%)$ & \\
\hline Female & $49(51.6 \%)$ & \\
\hline Age, years & $59.7 \pm 12.7$ & \\
\hline Duration of diabetes mellitus, years & $15.3 \pm 8.6$ & \\
\hline DM with HTN & $35(36.8 \%)$ & \\
\hline DM with CKD & $18(18.9 \%)$ & \\
\hline DM with Hyperlipidemia & $12(12.6 \%)$ & \\
\hline LogMAR BCVA at baseline & $0.540 \pm 0.355$ & \\
\hline Intraocular pressure, $\mathrm{mmHg}$ & $15.6 \pm 3.4$ & \\
\hline \multicolumn{3}{|l|}{ Lens status, n (\%) } \\
\hline Phakia & $113(76.9 \%)$ & \\
\hline Pseudophakia & $34(23.1 \%)$ & \\
\hline \multicolumn{3}{|l|}{ Stage of diabetic retinopathy, n (\%) } \\
\hline Moderate NPDR & $3(0.02 \%)$ & \\
\hline Severe NPDR & $89(60.5 \%)$ & \\
\hline PDR & $55(37.4 \%)$ & \\
\hline \multicolumn{3}{|l|}{ Type of diabetic macular edema in OCT, n (\%) } \\
\hline Diffuse macular edema & $48(32.7 \%)$ & \\
\hline Cystoids macular edema & $46(31.3 \%)$ & \\
\hline Serous retinal detachment & $53(36.0 \%)$ & \\
\hline Previous panretinal photocoagulation, n (\%) & $80(54.4 \%)$ & \\
\hline Follow up periods, months & $42.9 \pm 18.7$ & \\
\hline Mean number of intravitreal injection & $5.0 \pm 3.7$ & \\
\hline ERM group (total) & $7.1 \pm 4.8$ & \multirow{2}{*}{$0.007^{*}$} \\
\hline Non-ERM group (total) & $4.7 \pm 3.1$ & \\
\hline Incidence of ERM formation, $\mathrm{n}(\%)$ & $14(9.5 \%)$ & \\
\hline Mean time of ERM formation, months & $19.3 \pm 12.4$ & \\
\hline \multicolumn{3}{|l|}{ Anti-VEGF treatment, \% } \\
\hline ERM group & $53.8 \%$ & \multirow{2}{*}{$0.057^{*}$} \\
\hline Non-ERM group & $75.4 \%$ & \\
\hline
\end{tabular}

Table 1. Clinical characteristics of patients who received intravitreal injections for diabetic macular edema. Values are presented as the mean \pm standard deviations. DM: diabetes mellitus; HTN: hypertension; CKD: chronic kidney disease; BCVA: best-corrected visual acuity; ERM: epiretinal membrane; OCT: optical coherent tomography; NPDR: non-proliferative diabetic retinopathy; PDR: proliferative diabetic retinopathy; VEGF: vascular endothelial growth factor; ${ }^{*}$ Student t-test.

18 had chronic kidney disease, and 12 had hyperlipidemia. The mean logarithm of minimal angle of resolution (LogMAR) best corrected visual acuity (BCVA) was $0.540 \pm 0.355$, and the mean intraocular pressure was $15.6 \pm 3.4 \mathrm{mmHg}$ at the initial visit. Three eyes had moderate non-proliferative diabetic retinopathy (NPDR), 89 eyes had severe NPDR and 55 eyes had proliferative diabetic retinopathy (PDR). Moreover, 34 of 147 eyes were pseudophakic, and 80 eyes had received previous panretinal photocoagulation. The mean follow-up duration among all patients was $42.9 \pm 18.7$ months, and the mean number of intravitreal injections was $5.0 \pm 3.7$. The mean number of intravitreal injections among patients in the ERM group was $7.1 \pm 4.8$, which was significantly higher than $4.7 \pm 3.1$ for the non-ERM group $(P=0.007)$. The proportion of anti-VEGF intravitreal injections in the ERM group was $53.8 \%$, which was lower than the $75.4 \%$ for the non-ERM group; however, this difference was not statistically significant $(P=0.057)$.

Incidence of ERM and changes of BCVA and CMT. Fourteen eyes (9.5\%) developed secondary ERM during follow-up, and the mean time to ERM formation was $19.3 \pm 12.4$ months (Table 1). According to the intravitreal injection agent, 7 of 108 eyes (6.5\%) using anti-VEGF, 1 of 14 eyes (7.1\%) using triamcinolone, and 6 of 25 eyes $(24.0 \%)$ using dexamethasone implant developed secondary ERM. Compared to other agents, the incidence of ERM was significantly higher among patients who received dexamethasone implant injections $(P=0.025)$. Seven of 89 eyes (7.9\%) with severe NPDR and 7 of 55 eyes (12.7\%) with PDR developed secondary ERM and 8 of 80 eyes (10.0\%) who previously received panretinal photocoagulation also developed secondary ERM during follow-up periods. According to the type of DME in optical coherent tomography (OCT), 2 of 48 eyes (4.2\%) with diffuse 


\begin{tabular}{|c|c|c|}
\hline Incidence of ERM & Value & $P$ value \\
\hline \multicolumn{3}{|l|}{ According to drugs, n (\%) } \\
\hline Anti-VEGF & $7(6.5 \%)$ & \multirow{3}{*}{$0.025^{\dagger}$} \\
\hline Dexamethasone implant & $6(24.0 \%)$ & \\
\hline Triamcinolone & $1(7.1 \%)$ & \\
\hline \multicolumn{3}{|c|}{ Stage of diabetic retinopathy, n (\%) } \\
\hline Moderate NPDR & $0(0.0 \%)$ & \multirow{3}{*}{$0.539^{\dagger}$} \\
\hline Severe NPDR & $7(7.9 \%)$ & \\
\hline PDR & $7(12.7 \%)$ & \\
\hline \multicolumn{3}{|c|}{ History of panretinal photocoagulation, n (\%) } \\
\hline Yes & $8(10.0 \%)$ & \multirow{2}{*}{$0.790^{*}$} \\
\hline No & $6(9.0 \%)$ & \\
\hline \multicolumn{3}{|c|}{ Type of diabetic macular edema, $\mathrm{n}(\%)$} \\
\hline Diffuse macular edema & $2(4.2 \%)$ & \multirow{3}{*}{$0.077^{\dagger}$} \\
\hline Cystoids macular edema & $8(17.4 \%)$ & \\
\hline Serous retinal detachment & $4(7.5 \%)$ & \\
\hline
\end{tabular}

Table 2. The incidence of epiretinal membrane (ERM) formation according to clinical factors of diabetic retinopathy. ERM: epiretinal membrane; NPDR: non-proliferative diabetic retinopathy; VEGF: vascular endothelial growth factor; $*$ Student $t$-test, ${ }^{\dagger}$ One-way analysis of variance.

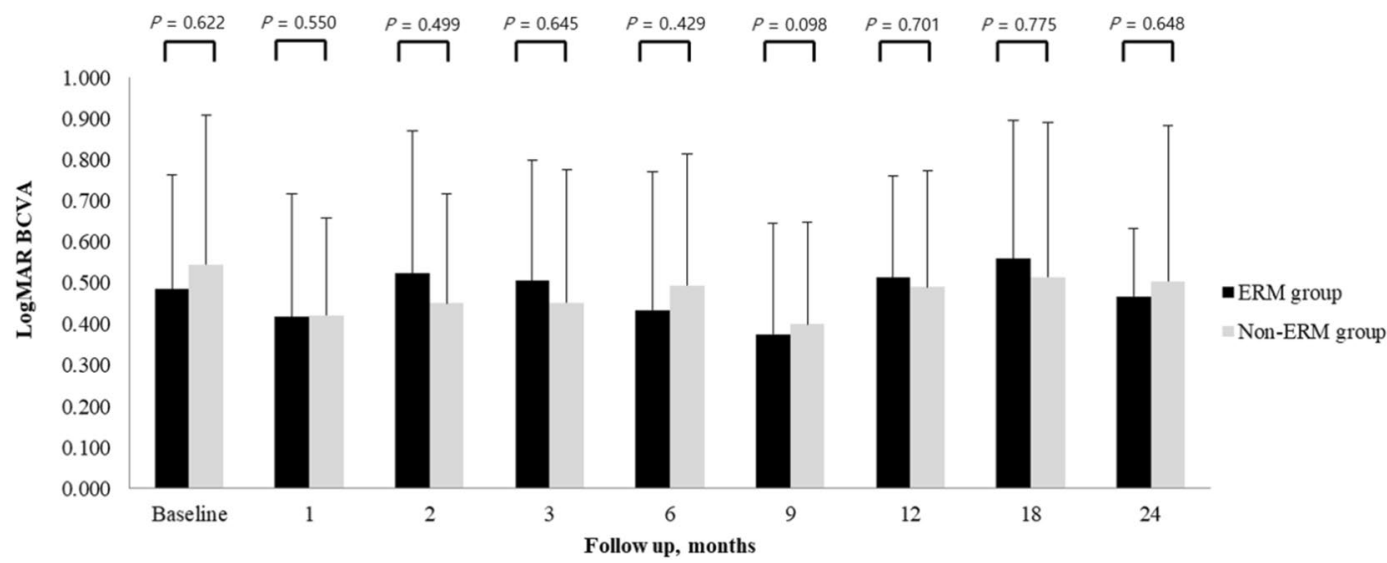

Figure 1. Mean best-corrected visual acuity (BCVA) changes during follow-up of the epiretinal membrane (ERM) and non-ERM groups (Student's t-test).

macular edema, 8 of 46 eyes (17.4\%) with cystoids macular edema, and 4 of 53 eyes (7.5\%) of DME with serous retinal detachment developed secondary ERM. Compared to other types, the incidence of ERM was higher among patients with cystoids macular edema, however, this difference was not statistically significant $(P=0.077)$ (Table 2).

The mean BCVA change was not different between the ERM and non-ERM groups, as shown in Fig. 1. Mean CMT significantly decreased from baseline in all patients at every follow-up visit. However, mean CMT of the ERM group was significantly thicker than that of the non-ERM group after 9 months until 24 months $(P<0.05$, respectively), as shown in Fig. 2.

In the ERM group, changes of BCVA from pre-injection to 2 weeks post-injection were not different before and after ERM formation, and changes of CMT from pre-injection to 2 weeks post-injection were significantly lower after ERM formation $(-62.0 \pm 60.9 \mu \mathrm{m})$ than before ERM formation $(-130.9 \pm 93.3 \mu \mathrm{m})(P=0.005)$, as shown in Table 3.

Clinical factors of epiretinal membrane formation. Table 4 shows the results of univariate and multivariate logistic regression analyses of associations between clinical parameters and secondary ERM formation. The number of intravitreal injections (odds ratio $[\mathrm{OR}]=1.212,95 \%$ confidence interval $[\mathrm{CI}]=1.047$ to 1.402 , $P=0.010)$ and the use of dexamethasone implant $(\mathrm{OR}=1.020,95 \% \mathrm{CI}=1.001$ to $1.040, P=0.041)$ were significantly associated with secondary ERM formation following intravitreal injection.

\section{Discussion}

It is well known that, among DME patients, there are high incidences of vitreomacular interface abnormality (VMIA), including ERM, vitreomacular attachment (VMA), and vitreomacular traction (VMT) with incomplete posterior vitreous detachment (PVD $)^{7,11-13}$. Although the prevalence of VMIA in DME patients has been shown to vary from $6.5 \%$ to $75 \%$ according to the diagnostic criteria and methods ${ }^{7,11,13}$, no information has been 


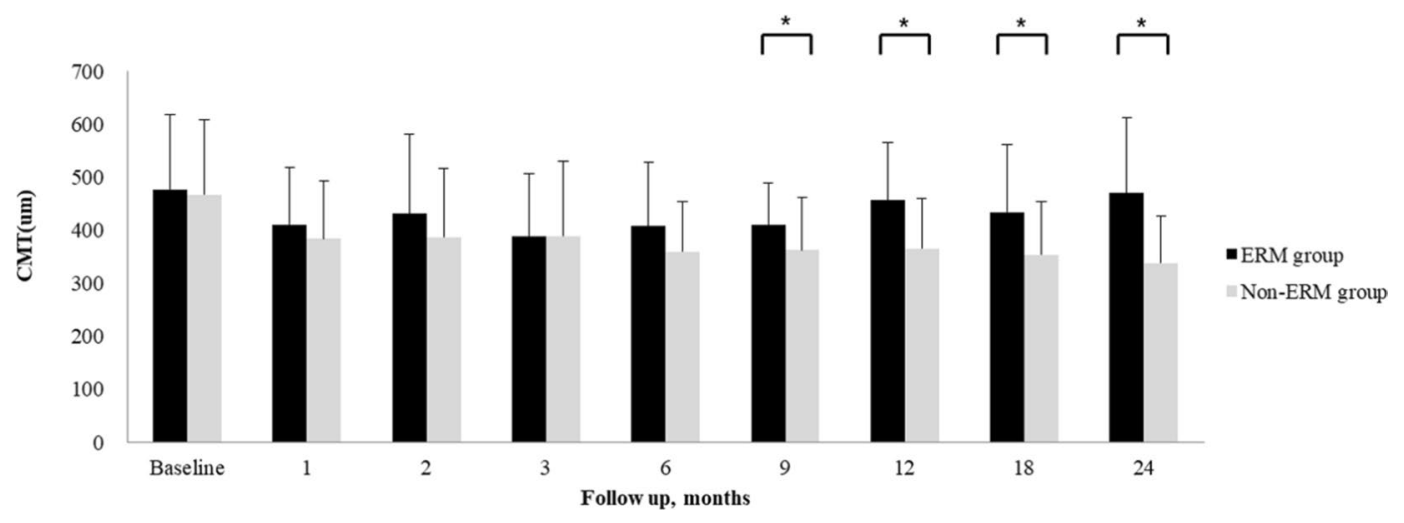

Figure 2. Mean central macular thickness (CMT) during follow-up of the epiretinal membrane (ERM) and non-ERM groups $(* P<0.05$, Student's t-test).

\begin{tabular}{|l|c|l|l|}
\hline & $\begin{array}{l}\text { Before ERM } \\
\text { formation }\end{array}$ & $\begin{array}{l}\text { After ERM } \\
\text { formation }\end{array}$ & P value \\
\hline LogMAR BCVA & $0.056 \pm 0.116$ & $0.031 \pm 0.104$ & 0.400 \\
\hline CMT $(\mu \mathrm{m})$ & $-130.9 \pm 93.3$ & $-62.0 \pm 60.9$ & $0.005^{*}$ \\
\hline
\end{tabular}

Table 3. Comparison of mean changes of BCVA and CMT after intravitreal injection before and after epiretinal membrane (ERM) formation in the ERM group. Values are presented as mean \pm standard deviation. BCVA: best-corrected visual acuity; CMT: central macular thickness. $* P<0.05$, Wilcoxon signed rank test.

published about secondary ERM formation after intravitreal injection for DME treatment. Thus, we performed this study specifically investigating secondary ERM among VMIAs based on OCT findings, and we found that 9.5\% of eyes developed secondary ERM after intravitreal injection.

The association between intravitreal injection and ERM formation is controversial. Chen et al. ${ }^{9}$ reported that platelet-derived growth factor (PDGF) A, VEGF, and their receptors are expressed in ERM and suggested these factors may contribute to ERM progression. They also reported that intravitreal injection of anti-VEGF agent might suppress VMIAs, including ERM formation. On the other hand, there have been reports of secondary ERM formation after intravitreal anti-VEGF injection ${ }^{14-16}$. Anti-VEGF has been reported to cause hypoxia in vascular endothelial cells and increase the expression of connective tissue growth factor (CTGF), which plays an important role in tissue fibrosis and ERM formation ${ }^{16}$. Moreover, Zhang et al. ${ }^{17}$ suggested that intravitreal bevacizumab (IVB) injection accelerated fibrosis in PDR patients via the upregulation of fibrosis-related cytokines and reported that IVB exerted pro-fibrotic effects after anti-VEGF therapy in vitro. In our study, $6.5 \%$ of the eyes that received intravitreal anti-VEGF injection developed secondary ERM, which may have been due to retinal tissue fibrosis after intravitreal anti-VEGF injection.

Currently, intravitreal injection of steroids is used to inhibit the production of inflammatory cytokines and VEGF gene expression in the management of $\mathrm{DME}^{18}$. Dexamethasone implant injection, a slow-releasing intravitreal delivery system, has a long-lasting beneficial effect in the treatment of DME. However, the incidence of secondary ERM was significantly higher after intravitreal dexamethasone implant injection than with other agents, and the use of dexamethasone implant is found to be a risk factor for ERM formation in this study. These results are thought to be associated with the drug delivery system. Unlike triamcinolone, dexamethasone implant containing a poly lactic-co-glycolic acid (PLGA) matrix was injected into the vitreous cavity using a special mechanical applicator. Meyer et al. ${ }^{19}$ reported that dexamethasone implant pellets have a significant muzzle velocity of approximately $0.8 \mathrm{~m} / \mathrm{s}$, which decreases exponentially over distance because of the drag force on the vitreous gel. In other words, intravitreal injection of dexamethasone implant may physically induce mechanical stress in the vitreous cavity, including the vitreomacular interface, and lead to VMIA ${ }^{20}$. In addition, it was reported that vitreoproliferative responses, including ERM formation, were observed after intravitreal implantation of a biodegradable PLGA, which comprises the drug vehicle for dexamethasone implant ${ }^{21}$. PLGA matrix that degrades to lactic acid and glycolic acid, and further degrade to carbon dioxide and water induced by hydrolysis ${ }^{22}$. Although, the safety of dexamethasone implant injection has been sufficiently verified, these processes could make alteration of vitreous structures including vitreous liquefaction, posterior vitreous detachment, and lead to vitreoproliferative response. Therefore, dexamethasone implant could be a risk factor of ERM formation after intravitreal injection.

In addition to dexamethasone implant, the analysis of relevant factors found that the incidence of ERM after intravitreal injection also increased as the number of intravitreal injections increased. It is well-known that intravitreal injection itself induces a $\mathrm{PVD}^{23}$ and that PVD is closely associated with ERM formation because glial cells and cortical vitreous remnants proliferate in the vitreomacular interface after the occurrence of PVD ${ }^{24}$. Therefore, we thought that frequent intravitreal injections could increase ERM incidence as a result of increased PVD occurrence, making the number of intravitreal injections a risk factor for ERM formation. 


\begin{tabular}{|c|c|c|c|c|c|c|}
\hline \multirow[b]{2}{*}{ Parameters } & \multicolumn{3}{|l|}{ Univariate } & \multicolumn{3}{|c|}{ Multivariate } \\
\hline & Odds ratio & CI (95\%) & $P$-value & Odds ratio & CI (95\%) & $P$-value \\
\hline Sex, male & 0.547 & 0.174 to 1.719 & 0.302 & & & \\
\hline Age at DM diagnosis & 1.033 & 0.984 to 1.084 & 0.193 & & & \\
\hline Duration of DM & 0.963 & 0.898 to 1.031 & 0.280 & & & \\
\hline Age at DME diagnosis & 1.012 & 0.967 to 1.058 & 0.608 & & & \\
\hline DM with CKD & 1.673 & 0.354 to 7.902 & 0.516 & & & \\
\hline DM with HTN & 3.975 & 0.855 to 18.480 & 0.078 & & & \\
\hline DM with Hyperlipidemia & 1.799 & 0.238 to 13.619 & 0.570 & & & \\
\hline Lens status, phakia & 1.373 & 0.402 to 4.693 & 0.613 & & & \\
\hline Refraction, S.E (Diopter) & 1.043 & 0.537 to 1.299 & 0.708 & & & \\
\hline LogMAR BCVA at initial visit & 0.470 & 0.080 to 2.747 & 0.402 & & & \\
\hline IOP at initial visit & 0.886 & 0.751 to 1.044 & 0.149 & & & \\
\hline Mean CMT at initial visit & 1.001 & 0.997 to 1.005 & 0.617 & & & \\
\hline PRP & 0.859 & 0.282 to 2.611 & 0.789 & & & \\
\hline Follow up periods & 1.007 & 0.979 to 1.036 & 0.619 & & & \\
\hline $\begin{array}{l}\text { Number of intravitreal } \\
\text { injections }\end{array}$ & 1.228 & 1.063 to 1.417 & 0.005 & 1.212 & 1.047 to 1.402 & 0.010 \\
\hline \multicolumn{7}{|l|}{ Agent of intravitreal injection } \\
\hline Anti-VEGF & 0.986 & 0.970 to 1.002 & 0.091 & & & \\
\hline Dexamethasone implant & 1.021 & 1.003 to 1.039 & 0.019 & 1.020 & 1.001 to 1.040 & 0.041 \\
\hline Triamcinolone & 0.996 & 0.972 to 1.020 & 0.735 & & & \\
\hline
\end{tabular}

Table 4. Univariate and multivariate analysis of risk factors of epiretinal membrane formation. BCVA: bestcorrected visual acuity; CMT: central macular thickness; CKD: chronic kidney disease; DM: diabetes mellitus; DME: diabetic macular edema; IOP: intraocular pressure; PRP: panretinal photocoagulation; S.E: spherical equivalent; VEGF: vascular endothelial growth factor.

We found that BCVA did not increase after ERM formation, and the change of CMT after ERM formation was significantly lower than before ERM formation after intravitreal injection for DME. This means that patients with secondary ERM showed a smaller decrease of CMT after intravitreal injection than before ERM formation, and the effect of intravitreal injection was reduced after ERM formation. This supported the previous report that ERM may act as a physical barrier and decrease drug penetration ${ }^{10}$.

There were several limitations to this study. First of all, we did not evaluate the status of PVD before and after intravitreal injection. However, Ophir et al..$^{25}$ previously reported that $52.2 \%$ of DME eyes with ERM had incomplete PVD on OCT, suggesting that ERM could appear when incomplete PVD occurred, and ERM could be possible even if complete PVD is not present. Moreover, this study was a retrospective chart review without a control group. This means that it was difficult to evaluate whether the ERM formation occurred as part of the natural course of DME or as a secondary effect after intravitreal injection. We measured BCVA using a Snellen chart and converted to a LogMAR scale instead of using the Early Treatment Diabetic Retinopathy Study (ETDRS) chart as a visual function test. We did not perform more comprehensive visual function tests, such as foveal sensitivity or contrast sensitivity testing. Despite these limitations, it is certain that intravitreal injection itself or dexamethasone implant injection is associated with secondary ERM formation, and the drug effect decreased after secondary ERM formation.

In conclusion, the effect of CMT reduction among DME patient with secondary ERM was significantly less than the effect among patients without ERM following intravitreal injection, and BCVA did not increase. Additionally, we found that the incidence of secondary ERM increased when dexamethasone implant was administered and as the number of intravitreal injections increased. Thus, patients should be informed that intravitreal injection for the treatment of DME may cause secondary ERM formation and that the effects of intravitreal injections may decline after ERM formation.

\section{Methods}

Medical records were retrospectively reviewed after approval of the Institutional Review Board of Kyungpook National University Hospital (IRB No. 2018-11-010). The review was conducted in accordance with the tenets of the Declaration of Helsinki. Written informed consent was obtained from all participants before study enrollment.

We included patients who had DR with DME aged over 18 years and who received at least 1 intravitreal injection of anti-VEGF, triamcinolone acetonide, or dexamethasone implant and were followed up for more than 24 months from January 2012 to December 2016. Patients received intravitreal injection of anti-VEGF or steroid followed a pro re nata (PRN) regimen, but ranibizumab, aflibercept and dexamethasone implant were injected according to health care insurance ${ }^{26-29}$. Patients were excluded if they met any of the following criteria: (1) the presence of uveitis or retinal vein occlusion that may cause macular edema; (2) history of procedures, such as neodymium:yttrium-aluminum-garnet (Nd:YAG) posterior capsulotomy or phacoemulsification that can cause macular edema within 1 month before intravitreal injection; (3) complications of DR other than DME, such as 
vitreous hemorrhage or tractional retinal detachment; (4) previous vitreoretinal surgery or glaucoma surgery; (5) the presence of media haze that may affect fundus examination and OCT examination; and (6) uncontrolled diabetes mellitus (serum HbAlc $>10 \%$ ).

DME was documented by fundus examination and spectral-domain OCT (SD-OCT, Spectralis ${ }^{\circledR}$, Heidelberg Engineering, Heidelberg, Germany). DME was defined as retinal thickening of more than 1 disc diameter, diffuse or cystoid changes within half of a disc diameter of the fovea and CMT $>300 \mu \mathrm{m}$ on OCT. The type of DME was classified as diffuse macular edema, cystoids macular edema and serous retinal detachment according to Otani et al..$^{30}$. ERM was defined as a highly reflective membranous structure at the vitreomacular interface on OCT ${ }^{24}$. OCT image interpretation including detection of ERM was performed by one physician (YKK).

All intravitreal injections were performed under sterile conditions in an operating room. Eyes were anesthetized with topical $0.5 \%$ proparacaine hydrochloride (Paracaine ${ }^{\circledR}$, Hanmi, Seoul, Korea), and $5 \%$ povidone-iodine was used for prophylaxis of endophthalmitis. Subsequently, intravitreal injection of an anti-VEGF agent, for example bevacizumab (Avastin ${ }^{\circledR}$, Genentech, South San Francisco, California, USA) $1.25 \mathrm{mg} / 0.05 \mathrm{~mL}$, ranibizumab (Lucentis ${ }^{\circledR}$, Genentech, South San Francisco, California, USA) $0.5 \mathrm{mg} / 0.05 \mathrm{~mL}$, or aflibercept (Eylea ${ }^{\circledR}$, Regeneron, Tarrytown, NY, USA) $2.0 \mathrm{mg} / 0.05 \mathrm{~mL}$ was administrated at 3.5 to $4.0 \mathrm{~mm}$ posterior to the limbus using a 30-gauge needle. Triamcinolone $\left(\right.$ Triam $^{\circledR}$, Shinpoong, Seoul, Korea) $4.0 \mathrm{mg} / 0.1 \mathrm{~mL}$ or dexamethasone implant (Ozurdex ${ }^{\circledR}$, Allergan, Irvine, CA, USA) were also administrated at the par plana. Topical 0.5\% levofloxa$\operatorname{cin}\left(\mathrm{Cravit}^{\circledR}\right.$, Santen, Osaka, Japan) eye drops were prescribed 4 times daily for 1 week after intravitreal injection.

Ophthalmic examinations were performed before and 2 weeks after every intravitreal injection, including BCVA using a Snellen chart, intraocular pressure (IOP), slit lamp examination, fundus examination, and SD-OCT examination. BCVAs were converted to the LogMAR for statistical analysis. All examinations were repeated after $1,2,3,6,9,12,18$, and 24 months after the first intravitreal injection. The number and type of intravitreal injection were obtained from operation records.

Statistical analyses were performed using SPSS Statistics version 20 (IBM Corp., Armonk, NY, USA). Repeated measures analysis of variance (ANOVA) corrected by the Bonferroni method was used to compare the mean BCVA and CMT according to the follow-up periods. Student's t-test was performed to compare the mean BCVA and CMT in both groups. In patients with secondary ERM, Student's t-test and one-way ANOVA were used to compare the incidence of ERM formation according to clinical factors. The Wilcoxon signed-rank test was used to compare BCVA and CMT changes between pre-injection and 2 weeks post-injection before and after ERM formation. Univariate logistic regression analysis was performed to calculate risk factors of secondary ERM. Variables with a $P$ value $<0.05$ in the univariate analysis were included in subsequent multivariate analysis. For all statistical tests, a $P$ value $<0.05$ was considered significant.

Received: 21 August 2019; Accepted: 2 January 2020;

Published online: 17 January 2020

\section{References}

1. Das, A., McGuire, P. G. \& Rangasamy, S. Diabetic Macular Edema: Pathophysiology and Novel Therapeutic Targets. Ophthalmology 122, 1375-1394, https://doi.org/10.1016/j.ophtha.2015.03.024 (2015).

2. Frank, R. N. Diabetic retinopathy. The New England journal of medicine 350, 48-58, https://doi.org/10.1056/NEJMra021678 (2004).

3. Rechtman, E., Harris, A., Garzozi, H. J. \& Ciulla, T. A. Pharmacologic therapies for diabetic retinopathy and diabetic macular edema. Clinical ophthalmology 1, 383-391 (2007).

4. Boyer, D. S. et al. Three-year, randomized, sham-controlled trial of dexamethasone intravitreal implant in patients with diabetic macular edema. Ophthalmology 121, 1904-1914, https://doi.org/10.1016/j.ophtha.2014.04.024 (2014).

5. Brown, D. M. et al. Intravitreal Aflibercept for Diabetic Macular Edema: 100-Week Results From the VISTA and VIVID Studies. Ophthalmology 122, 2044-2052, https://doi.org/10.1016/j.ophtha.2015.06.017 (2015).

6. Mitchell, P., Wong, T. Y. \& Diabetic Macular Edema Treatment Guideline Working, G. Management paradigms for diabetic macular edema. American journal of ophthalmology 157, 505-513 e501-508, https://doi.org/10.1016/j.ajo.2013.11.012 (2014).

7. Ghazi, N. G., Ciralsky, J. B., Shah, S. M., Campochiaro, P. A. \& Haller, J. A. Optical coherence tomography findings in persistent diabetic macular edema: the vitreomacular interface. American journal of ophthalmology 144, 747-754, https://doi.org/10.1016/j. ajo.2007.07.012 (2007).

8. Harada, C., Mitamura, Y. \& Harada, T. The role of cytokines and trophic factors in epiretinal membranes: involvement of signal transduction in glial cells. Progress in retinal and eye research 25, 149-164, https://doi.org/10.1016/j.preteyeres.2005.09.001 (2006).

9. Chen, Y. S. et al. Localisation of vascular endothelial growth factor and its receptors to cells of vascular and avascular epiretinal membranes. The British journal of ophthalmology 81, 919-926 (1997).

10. Ercalik, N. Y. et al. Influence of the epiretinal membrane on ranibizumab therapy outcomes in patients with diabetic macular edema. Arquivos brasileiros de oftalmologia 79, 373-375, https://doi.org/10.5935/0004-2749.20160106 (2016).

11. Ophir, A., Martinez, M. R., Mosqueda, P. \& Trevino, A. Vitreous traction and epiretinal membranes in diabetic macular oedema using spectral-domain optical coherence tomography. Eye 24, 1545-1553, https://doi.org/10.1038/eve.2010.80 (2010).

12. Gandorfer, A. et al. Epiretinal pathology of diffuse diabetic macular edema associated with vitreomacular traction. American journal of ophthalmology 139, 638-652, https://doi.org/10.1016/j.ajo.2004.11.035 (2005).

13. Kulikov, A. N. et al. Vitreoretinal interface abnormalities in diabetic macular edema and effectiveness of anti-VEGF therapy: an optical coherence tomography study. Clinical ophthalmology 11, 1995-2002, https://doi.org/10.2147/OPTH.S146019 (2017).

14. Abouammoh, M. A., Belliveau, M. J., Almeida, D. R., Gale, J. S. \& Sharma, S. Ranibizumab for idiopathic epiretinal membranes: A retrospective case series. Saudi journal of ophthalmology: official journal of the Saudi Ophthalmological Society 27, 79-82, https://doi. org/10.1016/j.sjopt.2013.01.002 (2013).

15. Cho, H. J. et al. Effect of Epiretinal Membranes on Antivascular Endothelial Growth Factor Treatment for Neovascular Age-Related Macular Degeneration. Journal of ocular pharmacology and therapeutics: the official journal of the Association for Ocular Pharmacology and Therapeutics 33, 452-458, https://doi.org/10.1089/jop.2016.0178 (2017).

16. Marticorena, J. et al. Intravitreal bevacizumab for retinal vein occlusion and early growth of epiretinal membrane: a possible secondary effect? The British journal of ophthalmology 95, 391-395, https://doi.org/10.1136/bjo.2009.177287 (2011).

17. Zhang, Q. et al. The relationship between anti-vascular endothelial growth factor and fibrosis in proliferative retinopathy: clinical and laboratory evidence. The British journal of ophthalmology 100, 1443-1450, https://doi.org/10.1136/bjophthalmol-2015-308199 (2016). 
18. Schwartz, S. G., Flynn, H. W., Jr. \& Scott, I. U. Intravitreal Corticosteroids in the Management of Diabetic Macular Edema. Current ophthalmology reports 1, https://doi.org/10.1007/s40135-013-0015-3 (2013).

19. Meyer, C. H. et al. Release and velocity of micronized dexamethasone implants with an intravitreal drug delivery system: kinematic analysis with a high-speed camera. Retina 32, 2133-2140, https://doi.org/10.1097/IAE.0b013e31825699e5 (2012).

20. Panjaphongse, R. \& Stewart, J. M. Vitreomacular Traction after Dexamethasone Intravitreal Implant (Ozurdex) Injection: The Effect of Anomalous Posterior Vitreous Detachment. Retinal cases \& brief reports 10, 55-57, https://doi.org/10.1097/ICB.0000000000000172 (2016).

21. Giordano, G. G., Chevez-Barrios, P., Refojo, M. F. \& Garcia, C. A. Biodegradation and tissue reaction to intravitreous biodegradable poly(D,L-lactic-co-glycolic)acid microspheres. Current eye research 14, 761-768, https://doi.org/10.3109/02713689508995797 (1995).

22. Gavini, E. et al. Biodegradable Microspheres as Intravitreal Delivery Systems for Prolonged Drug Release. What is their Eminence in the Nanoparticle Era? Current drug delivery 15, 930-940, https://doi.org/10.2174/1567201815666180226121020 (2018).

23. Geck, U. et al. Posterior vitreous detachment following intravitreal drug injection. Graefe's archive for clinical and experimental ophthalmology = Albrecht von Graefes Archiv fur klinische und experimentelle Ophthalmologie 251, 1691-1695, https://doi.org/10.1007/ s00417-013-2266-y (2013).

24. Stevenson, W., Prospero Ponce, C. M., Agarwal, D. R., Gelman, R. \& Christoforidis, J. B. Epiretinal membrane: optical coherence tomography-based diagnosis and classification. Clinical ophthalmology 10, 527-534, https://doi.org/10.2147/OPTH.S97722 (2016).

25. Ophir, A. \& Martinez, M. R. Epiretinal membranes and incomplete posterior vitreous detachment in diabetic macular edema, detected by spectral-domain optical coherence tomography. Invest Ophthalmol Vis Sci 52, 6414-6420, https://doi.org/10.1167/ iovs.10-6781 (2011).

26. Michaelides, M. et al. A prospective randomized trial of intravitreal bevacizumab or laser therapy in the management of diabetic macular edema (BOLT study) 12-month data: report 2. Ophthalmology 117, 1078-1086 e1072, https://doi.org/10.1016/j. ophtha.2010.03.045 (2010).

27. Do, D. V. et al. The DA VINCI Study: phase 2 primary results of VEGF Trap-Eye in patients with diabetic macular edema. Ophthalmology 118, 1819-1826, https://doi.org/10.1016/j.ophtha.2011.02.018 (2011).

28. Mitchell, P. et al. The RESTORE study: ranibizumab monotherapy or combined with laser versus laser monotherapy for diabetic macular edema. Ophthalmology 118, 615-625, https://doi.org/10.1016/j.ophtha.2011.01.031 (2011).

29. Panozzo, G., Gusson, E., Panozzo, G. \& Dalla Mura, G. Dexamethasone intravitreal implant for diabetic macular edema: indications for a PRN regimen of treatment. European journal of ophthalmology 25, 347-351, https://doi.org/10.5301/ejo.5000563 (2015).

30. Otani, T., Kishi, S. \& Maruyama, Y. Patterns of diabetic macular edema with optical coherence tomography. American journal of ophthalmology 127, 688-693, https://doi.org/10.1016/s0002-9394(99)00033-1 (1999).

\section{Author contributions}

Authors who contributed to research study design are as follows, Y.K.K., H.S.P., D.H.P., and J.P.S. Y.K.K. and J.P.S. drafted the manuscript and analyzed the data. H.S.P. and D.H.P. contributed to the interpretation of the results. All authors contributed to writing and editing of the manuscript.

\section{Competing interests}

The authors declare no competing interests.

\section{Additional information}

Correspondence and requests for materials should be addressed to J.P.S.

Reprints and permissions information is available at www.nature.com/reprints.

Publisher's note Springer Nature remains neutral with regard to jurisdictional claims in published maps and institutional affiliations.

Open Access This article is licensed under a Creative Commons Attribution 4.0 International License, which permits use, sharing, adaptation, distribution and reproduction in any medium or format, as long as you give appropriate credit to the original author(s) and the source, provide a link to the Creative Commons license, and indicate if changes were made. The images or other third party material in this article are included in the article's Creative Commons license, unless indicated otherwise in a credit line to the material. If material is not included in the article's Creative Commons license and your intended use is not permitted by statutory regulation or exceeds the permitted use, you will need to obtain permission directly from the copyright holder. To view a copy of this license, visit http://creativecommons.org/licenses/by/4.0/.

(c) The Author(s) 2020 